\title{
A Fuzzy Interpolation Cross-Correlation Method for Time-Delay Estimation of Partial Discharge UHF Pulse Signals
}

\author{
Bowen Guo, ${ }^{1}$ Songyuan Li, ${ }^{1}$ Qinghua Tang, ${ }^{1}$ Lin $\mathrm{Li}^{1}{ }^{1}$ and Pengfei Li $\mathbb{D}^{2}$ \\ ${ }^{1}$ Tianjin Power Supply Company of State Grid Corporation of China, Tianjin 300000, China \\ ${ }^{2}$ School of Electrical and Mechanical Engineering, Pingdingshan University, Pingdingshan 467000, China \\ Correspondence should be addressed to Pengfei Li; pengfei9966@126.com
}

Received 3 August 2021; Revised 6 October 2021; Accepted 8 October 2021; Published 23 October 2021

Academic Editor: Jit S. Mandeep

Copyright (c) 2021 Bowen Guo et al. This is an open access article distributed under the Creative Commons Attribution License, which permits unrestricted use, distribution, and reproduction in any medium, provided the original work is properly cited.

Time-delay estimation of partial discharge (PD) ultrahigh-frequency (UHF) pulse signals is one of the effective means to diagnose the local defect of electrical equipment. In order to improve the time-delay estimation accuracy of a multielement PD UHF pulse signal sensor array and reduce the cost of the PD detection system, a fuzzy interpolation cross-correlation method for time-delay estimation of PD UHF pulse signals was proposed in this paper. Improving the signal sampling rate is an effective way to improve the time-delay estimation accuracy. In this work, the fuzzy interpolation inference method was applied to interpolate intermediate values into the feature area of the PD UHF signal to improve the sampling rate, and then the cross-correlation method was used to estimate the time delay. This method not only improves the system sampling rate by fuzzy interpolation inference, which can make up for the deficiency of system equipment sampling performance, but also reduces the estimation error caused by noninteger multiple sampling intervals of signal time delay. The comparative experiment results demonstrate the power of the proposed method in improving the accuracy of the time-delay estimation of PD UHF pulse signals.

\section{Introduction}

Partial discharge (PD) can accelerate the insulation deterioration of electrical equipment, over time PD can lead to insulation failure, and then the electrical accident will happen [1]. PD detection and localization can help assess plant insulation conditions efficiently, and they have significant meaning to the safety and stability of electrical equipment [2].

PD ultrahigh-frequency (UHF) detection method has the advantages of strong anti-interference capability, high sensitivity, remote detection, and so on, and then it is widely used in electric equipment PD localization [3]. Recently, the PD UHF signal time-delay localization method has aroused researchers' great attention. The basic strategy is to use multiple antennas collecting PD UHF pulse signals in synchronization and calculate the time delay between the multiple collected PD UHF, and then the location of PD source can be located based on the known antennas coordinates and the time delay [4]. Therefore, the accuracy of time-delay estimation directly determines the locating accuracy. There are many approaches to estimating the time delay of the PD UHF signals, such as the threshold method, the peak method, the energy method, the wavelet transform method, and the cross-correlation method [5].

The threshold method sets the threshold of energy accumulation to estimate each PD UHF pulse signal arrival time and then be used to determine the time delay. The peak method detects the signal peak to determine the PD UHF pulse signal time delay [6]. Therefore, the threshold method and peak method are strongly influenced by noise and waveform distortion. The energy method estimates the time delay by taking the turning point of PD UHF signal accumulative energy [7]. Although the antinoise ability is increased, the selection of turning points is greatly affected by a human. The wavelet transform method can effectively detect the abrupt change points of signals and has high positioning accuracy [8]. However, due to the great influence of noise, it is easy to generate many false extreme points, resulting in location failure. The cross-correlation algorithm is simple in 
calculation and has strong antinoise ability, but it is restricted by the sampling rate of the system.

In order to obtain accurate signals time delay, a sampling rate up to tens of GHz is needed [9]. There are two groups of approaches to improving the PD UHF signal sampling rate. The first group of approaches focus on the hardware equipment and use a high-frequency digital oscilloscope to obtain the PD UHF signal [10]. The high cost and low utilization rates of detection equipment greatly restrict the popularization and application of these approaches. The second group of approaches uses a cubic spline interpolation algorithm to interpolate some values into the feature area of the UHF signal [11]. The limitation of this group of approaches is that the cubic spline cannot describe the PD UHF signal characteristics, and then it is difficult to improve the accuracy of the interpolated values.

Fuzzy interpolation inference is the process of formulating the mapping from an input scope to an output scope using fuzzy logic [12], which is a generalization of linear interpolation and can represent nonlinear, vague models, and high-dimensional systems using fuzzy rule bases representing the experts' knowledge [13]. Fuzzy interpolation inference has been widely and successfully applied to many fields, such as job shop scheduling [14] and robotic control [15], among others [16].

This work proposes a fuzzy interpolation inference cross-correlation method for the time-delay estimation of PD UHF pulse signals. This is implemented by applying the fuzzy interpolation inference, with the support of a crosscorrelation algorithm. Briefly, the transformation-based fuzzy rule interpolation (T-based FRI) is applied to evolve its rule base through the effective revision mechanism based on the high-frequency sampling PD UHF pulse data. The evolved rule bases which have the faculty for improving the accuracy of the interpolated values are used in fuzzy interpolation inference to improve the PD UHF pulse signals sampling rate in software and then reduce the frequency requirements of hardware equipment. In this way, the time delay is accurately estimated by the cross-correlation algorithm. The proposed fuzzy interpolation inference crosscorrelation method can improve the signals sampling rate by software, which makes up for the defect of the low sampling rate of the system hardware and reduces the estimation error caused by the noninteger multiple matching between the signal time delay and the sampling interval. The proposed method was validated and evaluated through an experiment implemented in the laboratory. The experiment results show that the proposed method has a high accuracy of time-delay estimation. The main contributions of this work are threefold: (1) proposing a fuzzy interpolation cross-correlation method for time-delay estimation of PD UHF pulse signals, (2) developing a fuzzy interpolation inference method for PD UHF pulse signals which was used to improve the sampling rate in software, and (3) validating and evaluating the proposed method by a PD experiment.

The rest of the paper is organized as follows. Section 2 details the proposed fuzzy interpolation inference crosscorrelation method. Section 3 applies the proposed method to PD UHF pulse signals time-delay estimation in the laboratory and verified the effectiveness. The paper is concluded in Section 4 and the possible further work is pointed out.

\section{Methods}

The proposed fuzzy interpolation inference cross-correlation method is used to estimate time delay of PD UHF pulse signals through fuzzy interpolation approach to improve the sampling rate of UHF signal and then through the crosscorrelation function to estimate the time delay. Accordingly, the proposed method consists of two key subsystems, including the fuzzy interpolation inference for PD UHF pulse signals and the cross-correlation algorithm for time-delay estimation.

2.1. Fuzzy Interpolation Inference for PD UHF Pulse Signals. Fuzzy interpolation inference is a generalization of crisp linear interpolation under the uncertainty representation framework of fuzzy logic [17]. In this work, fuzzy interpolation inference is used to interpolate some values into the collected PD UHF signal with a low sample rate, and it is a key component in the proposed method for the time-delay estimation of PD UHF pulse signals.

The framework of the fuzzy interpolation inference is shown in Figure 1 which comprises four main parts: (1) rule base initialization, (2) rule selection, (3) fuzzy rule interpolation, and (4) rule base revision. In particular, the rule base is initialized with experts' knowledge of PD UHF pulse signals. The transformation-based fuzzy rule interpolation (T-Based FRI) is particularly applied in this work to perform FRI inference due to its effectiveness for interpolating value estimation $[15,16]$. Rule base revision is used to generate an excellent rule base. Note that the rule base will not be revised after the completion of training; that is to say, the excellent rule base will be used for the interpolation without revision.

2.1.1. Rule Base Initialization. Fuzzy interpolation inference can be inferred by fuzzy interpolation based on finite fuzzy rules. Theoretically speaking, fuzzy interpolation inference can be used to infer any decision requirement based on system input through fuzzy interpolation under the condition of only two fuzzy rules. In this paper, the rule base is initialized with experts' knowledge of the PD UHF pulse signals. Each rule in the rule base should be two-input and one-output rule which is of the following format:

$$
R_{i}: \text { IF } x_{1} \text { is } A_{i 1} \text { AND } x_{2} \text { is } A_{i 2} \text {, THEN } y \text { is } B_{i} \text {, }
$$

where $A_{i 1}, A_{i 2}$, and $B_{1}$ are fuzzy sets, $x_{1}$ and $x_{2}$ represent the value of two consecutive PD HUF pulse signals, and the output $y$ is the interpolation value. For simplicity, triangular membership functions are employed in this work to represent the fuzzy sets. It should be noted that the initialized rule base should be interpolated and revised for good work.

2.1.2. Rule Selection. In order to enable the operation of fuzzy interpolation inference, two rules should be selected for interpolation. In this work, the selected rules 


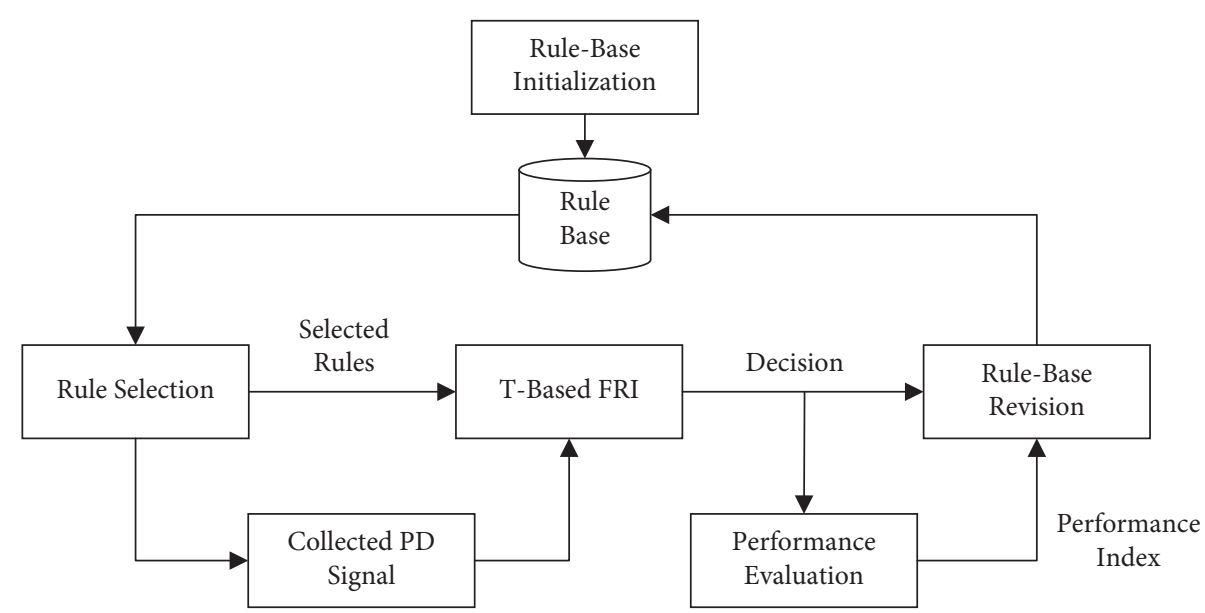

FIGURE 1: The framework of fuzzy interpolation inference for PD UHF signals.

for interpolation are based on the Euclidean distance between the given input and rule antecedents. The neighboring sampled PD UHF pulse signals $x_{1}^{*}$ and $x_{2}^{*}$ are represented by triangular fuzzy sets $A_{1}^{*}\left(a_{11}^{*}, a_{12}^{*}, a_{13}^{*}\right)$ and $A_{2}^{*}\left(a_{21}^{*}, a_{22}^{*}, a_{23}^{*}\right)$ and used as the given observations. Suppose the interpolation rule is expressed as

$$
R^{*}: \text { IF } x_{1} \text { is } A_{1}^{*} \text { AND } x_{2} \text { is } A_{2}^{*} \text {, THEN } y \text { is } B^{*} \text {. }
$$

The triangular fuzzy sets $A_{1}^{*}$ and $A_{2}^{*}$ can be given as

$$
\left\{\begin{array}{l}
x_{1}^{*}=\frac{a_{11}^{*}+a_{12}^{*}+a_{13}^{*}}{3}, \\
x_{2}^{*}=\frac{a_{21}^{*}+a_{22}^{*}+a_{23}^{*}}{3}, \\
a_{11}^{*} \leq a_{12}^{*} \leq a_{13}^{*}, \\
a_{21}^{*} \leq a_{22}^{*} \leq a_{23}^{*} .
\end{array}\right.
$$

For the convenience of fuzzy interpolation, the representative value of the fuzzy set is used, which is defined as follows:

$$
\operatorname{Rep}(A)=\frac{a_{1}+a_{2}+a_{3}}{3} .
$$

In this work, the two closest rules based on the Euclidean distance are selected for interpolation. Suppose the $i$-th rule in the rule bases is shown in equation (1). The distance between the interpolation rule and the $i$-th rule is as follows:

$$
D_{i}=\sqrt{\left(\frac{\left|\operatorname{Rep}\left(A_{1}^{*}\right)-\operatorname{Rep}\left(A_{1 i}\right)\right|}{\max \left(A_{1}^{*}\right)-\min \left(A_{1 i}\right)}\right)^{2}+\left(\frac{\left|\operatorname{Rep}\left(A_{2}^{*}\right)-\operatorname{Rep}\left(A_{2 i}\right)\right|}{\max \left(A_{2}^{*}\right)-\min \left(A_{2 i}\right)}\right)^{2}} .
$$

After traversing all rules in the current fuzzy rule base, the two closest rules are selected to perform the fuzzy interpolation operation.
2.1.3. Fuzzy Rule Interpolation. Given the two consecutive sampled PD UHF pulse signals $x_{1}^{*}$ and $x_{2}^{*}$, the two closest rules are selected to support the interpolation inference. Suppose that the selected two rules for interpolation are $R_{i}$ and $R_{j}$ and the form is expressed in equation (1). In this work, the scale and move transformation-based approach is used for interpolation inference; the process is summarized in the following steps.

Step 1. Calculate the relative placement factor $\lambda$.

The relative placement factor represents the location relationship between the current two consecutive sampled PD UHF signals and the two selected fuzzy rules. Then, the relative placement factor can be calculated as follows:

$$
\lambda_{n}=\frac{d\left(\operatorname{Rep}\left(A_{n i}\right), \operatorname{Rep}\left(A_{n}^{*}\right)\right)}{d\left(\operatorname{Rep}\left(A_{n i}\right), \operatorname{Rep}\left(A_{n j}\right)\right)}, \quad n=1,2
$$

The weighted average of $\lambda_{1}$ and $\lambda_{2}$ is taken as the relative placement factor $\lambda$ of the current decision input with the two selected rules, which can be expressed as

$$
\lambda=\frac{1}{2}\left(\lambda_{1}+\lambda_{2}\right)
$$

Step 2. Obtain the new intermediate rule $R^{*^{\prime}}$.

The new intermediate rule $R^{*^{\prime}}$ can be expressed as

$$
R^{*^{\prime}}: \text { IF } x_{1} \text { is } A_{1}^{*^{\prime}} \text { AND } x_{2} \text { is } A_{2}^{*^{\prime}} \text {, THEN } y \text { is } B^{*^{\prime}} \text {. }
$$

By using the simplest linear interpolation, the fuzzy sets of the intermediate rule can be calculated by

$$
\left\{\begin{array}{l}
A_{1}^{*^{\prime}}=\left(1-\lambda_{1}\right) A_{1 i}+\lambda_{1} A_{1 j}, \\
A_{2}^{*^{\prime}}=\left(1-\lambda_{2}\right) A_{2 i}+\lambda_{2} A_{2 j}, \\
B^{*^{\prime}}=(1-\lambda) B_{i}+\lambda B_{j} .
\end{array}\right.
$$

Step 3. Obtain the scaling rate. 
By comparing the antecedent of the intermediate rule and the current decision input, the scale rate can be obtained. The scaling rate of $A_{1}^{*}$ and $A_{1}^{*}$ which can be expressed as $s_{1}$ can be calculated using the following equation:

$$
s_{1}=\frac{a_{13}^{*^{\prime}}-a_{11}^{*^{\prime}}}{a_{13}^{*}-a_{11}^{*}}
$$

The scaling rate between $A_{2}^{*}$ and $A_{2}^{*}$ which can be represented as $s_{2}$ can be calculated using the same operation with $s_{1}$ (equation (10)).

Since the fuzzy rule involves two decision inputs, $s_{1}$ and $s_{2}$, the weighted average can be taken as the transformation scaling factor $(S)$ of the intermediate rule and the current decision input, which can be expressed as

$$
S=\frac{1}{2}\left(s_{1}+s_{2}\right)
$$

Step 4. Obtain the second intermediate rule $R^{*^{\prime \prime}}$.

The second intermediate rule $R^{*^{\prime \prime}}$ can be expressed as

$$
R^{* \prime \prime}: \text { IF } x_{1} \text { is } A_{1}^{*} \text { AND } x_{2} \text { is } A_{2}^{*} \text {, THEN } y \text { is } B^{* \prime \prime} \text {. }
$$

Using the scaling rate to obtain the second intermediate rule, then the fuzzy set $A_{1}^{*}$ " of the rule can be calculated as

$$
\left\{\begin{array}{l}
a_{11}^{*^{\prime \prime}}=\frac{a_{11}^{*^{\prime}}\left(1+2 s_{1}\right)+a_{12}^{*^{\prime}}\left(1-s_{1}\right)+a_{13}^{*^{\prime}}\left(1-s_{1}\right)}{3} \\
a_{12}^{*^{\prime \prime}}=\frac{a_{11}^{*^{\prime}}\left(1-s_{1}\right)+a_{12}^{*^{\prime}}\left(1+2 s_{1}\right)+a_{13}^{*^{\prime}}\left(1-s_{1}\right)}{3} \\
a_{13}^{*^{\prime \prime}}=\frac{a_{11}^{*^{\prime}}\left(1-s_{1}\right)+a_{12}^{*^{\prime}}\left(1-s_{1}\right)+a_{13}^{*^{\prime}}\left(1+2 s_{1}\right)}{3}
\end{array}\right.
$$

The fuzzy sets $A_{2}^{*}$ " and $B^{* \prime \prime}$ can be obtained using the same operation with $s_{2}$ and $S$ (equation (13)).

Step 5. Obtain the moving transformation rate.

By comparing the antecedent of the intermediate rule and the current decision input, the moving transformation rate can be obtained. The moving transformation rate of $A_{1}^{*}$ and $A_{1}^{*}$ " which can be expressed as $m_{1}$ can be calculated using the following equation:

$$
m_{1}= \begin{cases}\frac{3\left(a_{11}^{*}-a_{11}^{*^{\prime \prime}}\right)}{a_{12}^{*^{\prime \prime}}-a_{11}^{*^{\prime \prime}}}, & a_{11}^{*} \geq a_{11}^{*^{\prime \prime}}, \\ \frac{3\left(a_{11}^{*}-a_{11}^{*^{\prime \prime}}\right)}{a_{13}^{*^{\prime \prime}}-a_{12}^{*^{\prime \prime}}}, & a_{11}^{*}<a_{11}^{*^{\prime \prime}} .\end{cases}
$$

The moving transformation rate of $A_{2}^{*}$ and $A_{2}^{*}$ " represented by $m_{2}$ can be obtained using the same operation with $m_{1}$. The moving transformation rate $(M)$ is calculated using the weighted average of $m_{1}$ and $m_{2}$ and can be expressed as

$$
M=\frac{1}{2}\left(m_{1}+m_{2}\right)
$$

Step 6. Obtain the interpolation rule consequence $B^{*}$.

Firstly, the translation distance $l$ is calculated according to the moving transformation rate $M$ as follows:

$$
l= \begin{cases}M \frac{b_{2}^{*^{\prime \prime}}-b_{1}^{*^{\prime \prime}}}{3}, & M \geq 0, \\ M \frac{b_{3}^{*^{\prime \prime}}-b_{2}^{*^{\prime \prime}}}{3}, & M<0 .\end{cases}
$$

Secondly, the translation distance $l$ is calculated according to the interpolation rule consequence $B^{*}$, using the following equation:

$$
\left\{\begin{array}{l}
b_{1}^{*}=b_{1}^{*^{\prime \prime}}+l, \\
b_{2}^{*}=b_{2}^{*^{\prime \prime}}-2 l, \\
b_{3}^{*}=b_{3}^{*^{\prime \prime}}+l .
\end{array}\right.
$$

The representative value of the interpolation rule consequence $B^{*}$ is the current interpolation inference decision $y=\operatorname{Rep}\left(B^{*}\right)$; that is, $y$ is the interpolation data of two consecutive sampled PD UHF pulse signals.

2.1.4. Rule Base Revision. The high-frequency sampling PD data are used to train the fuzzy rule base. The sampled data are divided into label data $A$ and $B$ by taking one datum at an interval of one sampling point. The label data $A$ is the given input, and the label data $B$ is the given output for training the fuzzy rule base. In order to evaluate the performance of the interpolation rule, the performance index is used to evaluate the performance of the interpolation rule which indicates the difference between the interpolation data and the given output.

In order to avoid redundant or duplicated rules, the similarity degree $(S)$ between the interpolated rule and each rule in the existing rule base is calculated. Suppose that the interpolated rule is $R^{*}$ and the existing rule is $R_{i}$. The similarity degree between the two rules is computed as

$$
S_{i}=\frac{S\left(A_{i 1}, A_{1}^{*}\right)+S\left(A_{i 2}, A_{2}^{*}\right)+S\left(B_{i}, B^{*}\right)}{3},
$$

where $S(\bullet)$ represents the similarity degree calculation between two fuzzy sets and the fuzzy sets $A^{*}$ and $A_{i}$ can be expressed as follows:

$$
S\left(A_{i}, A^{*}\right)=1-\frac{\left|a_{i 1}-a_{1}^{*}\right|+\left|a_{i 2}-a_{2}^{*}\right|+\left|a_{i 3}-a_{3}^{*}\right|}{3\left|a_{i 1}-a_{i 3}\right|} .
$$

When a new interpolated rule $R^{*}$ is generated, the performance index should be calculated first. If the performance index meets the system requirements, then the similarity degree between the interpolated rule and each rule in the existing rule base should be calculated. Based on a predefined similarity degree threshold, if there is no similar rule in the existing rule 
base, the interpolated rule will be added to the rule base; if there are similar rules in the existing rule base, the best performance index rules will be preserved in the rule base.

After the rule base completion training, an excellent rule base will be generated which can express the PD feature information. And then, based on the excellent rule base, the PD UHF pulse signals with a low sample rate could process the interpolation inference to obtain the high sampling rate data.

\subsection{Cross-Correlation Algorithm for Time-Delay Estimation.} Cross-correlation is a basic method to measure the similarity degree of two signals in the time domain and is used to describe the related degree of the two signals in any two different times $t_{1}$ and $t_{2}$. Therefore, in this work, the crosscorrelation function is used to estimate the time delay of the received two PD UHF pulse signals $x_{1}(t)$ and $x_{2}(t)$ which can be expressed as follows:

$$
\left\{\begin{array}{l}
x_{1}(t)=A_{1} s\left(t-\tau_{1}\right)+n_{1}(t), \\
x_{2}(t)=A_{2} s\left(t-\tau_{2}\right)+n_{2}(t),
\end{array}\right.
$$

where $A_{1}$ and $A_{2}$ denote the amplitude gain of the two signals, $n_{1}(t)$ and $n_{2}(t)$ represent the unknown white Gaussian noise, and $\tau_{1}$ and $\tau_{2}$ are the signal-propagation time delay $\left(\tau_{1} \leq \tau_{2}\right)$. In order to express convenience, with $x_{1}(t)$ being a standard for normalization, equation (20) can be rewritten as

$$
\left\{\begin{array}{l}
x_{1}(t)=s(t)+n_{1}(t), \\
x_{2}(t)=\rho s(t-D)+n_{2}(t),
\end{array}\right.
$$

where $\rho=A_{1} / A_{2}$ represents the ratio of two-signal amplitude gains and $D=\tau_{2}-\tau_{1}$ denotes the time delay. The cross-correlation function of the two PD UHF pulse signals can be expressed as follows:

$$
\begin{aligned}
R_{12}(\tau) & =E\left[x_{1}(t) \cdot x_{2}(t+\tau)\right] \\
& =E\left\{\left[s(t)+n_{1}(t)\right] \cdot\left[\lambda s(t+\tau-D)+n_{2}(t+\tau)\right]\right\} \\
& =\lambda R_{s s}(\tau-D)+R_{s n 2}(\tau)+\lambda R_{n 1 s}(\tau-D)+R_{n 1 n 2}(\tau) .
\end{aligned}
$$

$n_{1}(t)$ and $n_{2}(t)$ are the Gaussian white noises independent of the PD UHF pulse signals, and the existing $R_{n 1 s}(\tau-D)=0, R_{s n 2}(\tau)=0$, and $R_{\mathrm{n} 1 \mathrm{n} 2}(\tau)=0$. And then the following equation holds:

$$
R_{12}(\tau)=\lambda R_{s s}(\tau-D)
$$

The cross-correlation function is subject to $\left|R_{s s}(\tau)\right| \leq\left|R_{s s}(0)\right|$, and $R_{12}(\tau)$ is maximized as $\tau=D$. Therefore, as the cross-correlation is peak $R_{12}(\tau)$, the corresponding time $\tau$ is the time delay for the two PD UHF pulse signals.

\section{Partial Discharge Experiment Study}

3.1. Equipment Arrangement. The proposed method was applied to a PD UHF pulse signal time-delay estimation experiment in the laboratory for method validation and evaluation. The equipment arrangement of the measurement is shown in Figure 2.
The simulated PD source consisted of a pair of pointto-point electrodes with an air gap and a Tesla coil. The directional antenna was used to obtain the PD UHF pulse signals, the bandwidth is $0.5 \sim 3 \mathrm{GHz}$, and the maximum gain is $7.9 \mathrm{dBi}$. The distance between the two antennas is $1.5 \mathrm{~m}$. The LeCroy WR640Zi oscilloscope was used to acquire signals with a bandwidth of $4 \mathrm{GHz}$ and a maximum sampling rate of $40 \mathrm{GS} / \mathrm{s}$. The two antennas were connected to the oscilloscope through identical coaxial cables.

The height of the antennas is regarded as the xoz plane, the vertical direction is the $z$-axis, and then the established coordinate system is shown in Figure 3. In this coordinate system, the coordinate of the PD source is $P(1.2,2.8,0.8)$, and the two antennas were placed at $A(-1.1,0,0)$ and $B(1.1,0,0)$.

3.2. Rule Base Construction. The fuzzy interpolation inference mechanism is detailed in Section 2.1, and thus the focus of this section is on the construction and evolving of the fuzzy rule base. As detailed in Section 2.1.4, the fuzzy rule base is constructed and evolved with the support of the highfrequency sampling PD UHF pulse signal data. According to the experts' knowledge, three initial rules were initialized firstly, and the fuzzy sets in initialized rule base are summarized in Table 1.

Based on this initialized rule base, the proposed system can perform fuzzy interpolation inference to interpolate some values into the received PD UHF signal with a low sampling rate. Note that the simple initialized rule base is not able to produce accurate performance, but the fuzzy rule base revision mechanism is able to evolve the rule base while it performs better performance. The process of fuzzy rule base revision is summarized as follows:

Step 1: fuzzily the given neighboring sampled PD UHF pulse signals as the fuzzy sets $A_{1}^{*}=(1.50,1.70,2.00)$ and $A_{2}^{*}=(1.80,2.10,2.50)$.

Step 2: select the two closest rules in the fuzzy rule base for interpolation using equation (5). For this case, the rules $R_{1}$ and $R_{2}$ were selected.

Step 3: interpolate the fuzzy rule. In this case, the parameters in this step are the relative placement factor $\lambda=0.775$, the move rate $M=-0.21$, and the scale rate $S=0.658$. From this, the resulting interpolated rule is "IF $x_{1}$ is $(1.50,1.70,2.00)$ AND $x_{2}$ is $(1.80,2.10,2.50)$, THEN $y$ is $(1.62,2.03,2.31)$ ".

Step 4: defuzzify the interpolated fuzzy rule consequence $B^{*}=(1.62,2.03,2.31)$ to the crisp value 1.99 , which was the interpolation inference decision.

Step 5: calculate performance index based on the interpolation inference decision and the given output. As the interpolation inference decision is equal to the desired output, a positive performance index is returned.

Step 6: revise the rule base based on the performance index feedback. In this case, the feedback was positive. Thus, the similarity degrees between the interpolated rule and each rule in the existing rule base should be 


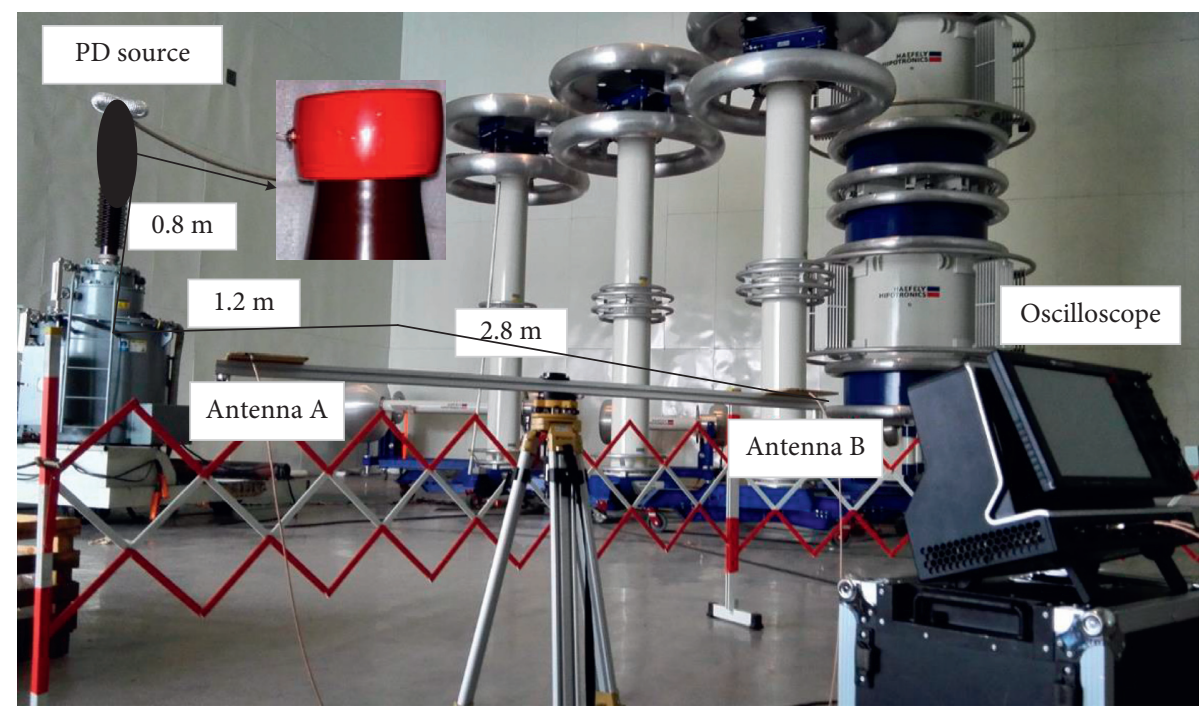

FIgURE 2: The equipment arrangement of the measurement.

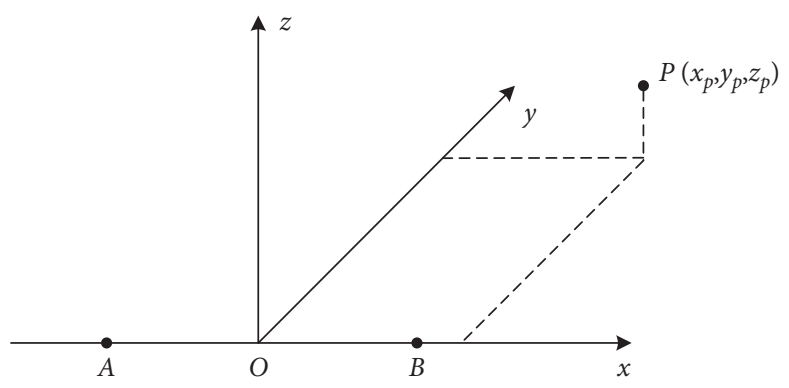

FIgURE 3: The established coordinate system of the equipment arrangement.

TABLE 1: The fuzzy sets in the initialized rule base.

\begin{tabular}{cccc}
\hline$i$ & $A_{i 1}$ & $A_{i 2}$ & $B_{i}$ \\
\hline 1 & $(2.02,2.51,3.03)$ & $(4.52,5.04,6.01)$ & $(3.21,3.72,4.24)$ \\
2 & $(1.01,1.21,1.58)$ & $(1.51,1.91,2.35)$ & $(1.21,1.49,2.03)$ \\
3 & $(-3.02,-2.53$, & $(-2.17,-1.79$, & $(-2.61,-2.14,-1.85)$ \\
& $-2.21)$ & $-1.31)$ & \\
\hline
\end{tabular}

calculated. The maximum similarity degree was 0.47 , which is smaller than the predefined threshold of 0.7 . Therefore, the interpolated rule is added to the existing rule base as follows:

$$
R^{*}: \text { IF } x_{1} \text { is } A_{1}^{*} \text { AND } x_{2} \text { is } A_{2}^{*} \text {, THEN } y \text { is } B^{*} \text {. }
$$

The above process is repeated for every new input, and it will be stabilized after 3000 inference iterations. A random snapshot of the rule base is summarized in Table 2 .

3.3. Experiment Result Analysis. In this section, the PD UHF pulse signals are collected with $1 \mathrm{GHz}$ sampling rate. The equipment arrangement of the measurement and the coordinate system is shown in Figures 2 and 3. Using their experiment equipment, the collected PD UHF pulse signals are shown in Figures 4(a) and 4(b). From this figure, it is clear that the wavefront of the two PD UHF pulse signals is similar. This is because the two signals are coming from the same source.

Based on the evolved fuzzy rule base, the fuzzy interpolation inference is used to interpolate intermediate values into the collected PD UHF pulse signals. The originally collected PD UHF pulse signals are interpolated by 1 point, 3 points, 7 points, and 15 points in adjacent sampling points, respectively. That is, the sampling rates after interpolation are $2 \mathrm{GHz}, 4 \mathrm{GHz}, 8 \mathrm{GHz}$, and $16 \mathrm{GHz}$, respectively.

As the cubic spline interpolation has good convergence and stability, it is often used in numerical interpolation operations. In this paper, the cubic spline interpolation is used as a comparison to verify the effectiveness of the proposed method. The wavefront of the PD UHF pulse signals is in the range of $30 \mathrm{~ns}$ to $80 \mathrm{~ns}$ in this experiment, so only the signals within this interval are analyzed. The interpolated $2 \mathrm{GHz} \mathrm{PD}$ UHF pulse signals are shown in Figures 5(a) 5(d). These figures show that the interpolated $2 \mathrm{GHz}$ PD UHF pulse signals curved by fuzzy interpolation are smoother than cubic spline interpolation. The interpolated $4 \mathrm{GHz}$ and $8 \mathrm{GHz} \mathrm{PD}$ UHF pulse signals have similar performance and are omitted here to save space. The correlation coefficient curve is shown in Figures 5(e) 5(f), and the maximum correlation coefficient 
TABLE 2: The fuzzy sets in the initialized rule base.

\begin{tabular}{lccr}
\hline$i$ & $A_{i 1}$ & $A_{i 2}$ & $B_{i}$ \\
\hline 1 & $(12.31,12,74,13.25)$ & $(14.53,15.02,15.47)$ & $(13.44,13.91,14.35)$ \\
2 & $(8.47,8.82,9.53)$ & $(8.91,9.43,9.92)$ & $(8.71,9.14,9.37)$ \\
3 & $(4.52,4.95,5.61)$ & $(6.17,6.52,6.94)$ & $(5.33,5.72,6.27)$ \\
4 & $(2.01,2,53,3.02)$ & $(4.54,5.01,6.07)$ & $(3.23,3.71,3.28)$ \\
5 & $(-3.06,-2.51,-2.27)$ & $(-2.13,-1.78,-1.31)$ & $(-2.63,-2.15,-1.87)$ \\
6 & $(-6.06,-5,63,-5.07)$ & $(-5.41,-4.94,-4.42)$ & $(-5.73,-5.31,-4.76)$ \\
7 & $(-9.25,-8.83,-8.35)$ & $(-8.96,-8.32,-7.97)$ & $(-9.12,-8.62,-8.17)$ \\
8 & $(-12.32,-11.84,-11.21)$ & $(-11.74,-11.17,-10.82)$ & $(-12.02,-11.56,-11.01)$ \\
\hline
\end{tabular}

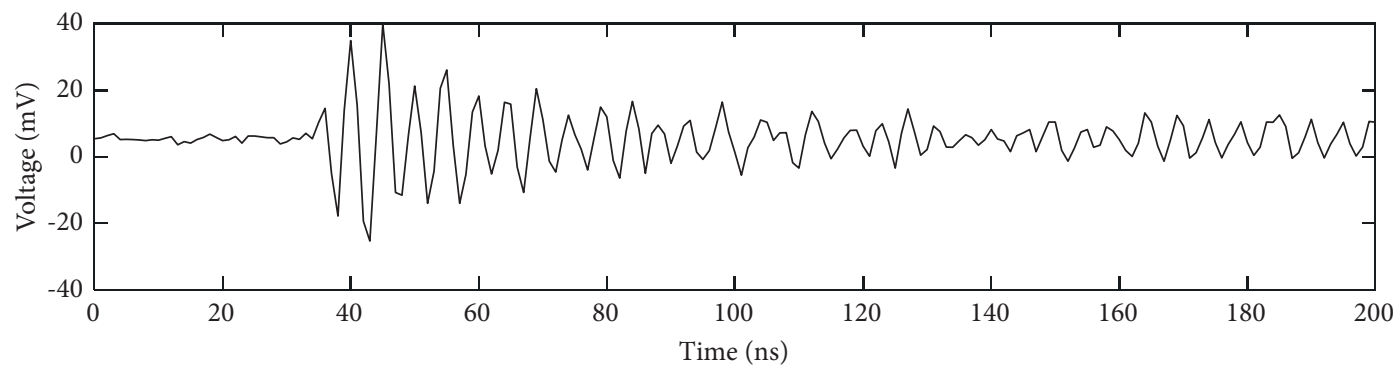

(a)

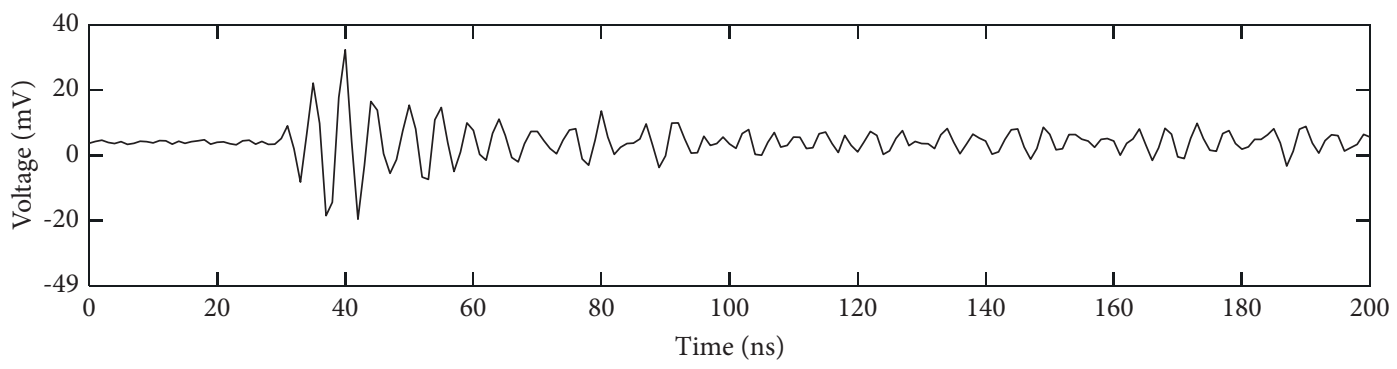

(b)

Figure 4: The collected PD UHF pulse signals with a $1 \mathrm{GHz}$ sampling rate. (a) Antenna A received PD UHF pulse signals. (b) Antenna B received $\mathrm{PD}$ UHF pulse signal.

happens as 7 translation points and 8 translation points, respectively. That is, the calculated time delays are $3.5 \mathrm{~ns}$ and 4 ns for fuzzy interpolation inference and cubic spline interpolation, respectively.

The propagation speed of the PD UHF pulse signals in the air is about $3 \times 10^{8} \mathrm{~m} / \mathrm{s}$, and according to the coordinate information, the straight-line distance between the PD source and the two antennas is $3.71 \mathrm{~m}$ and $2.91 \mathrm{~m}$, respectively. The difference between the two straight-line distances is $0.8 \mathrm{~m}$. Therefore, the time delay between two PD signal receiving channels should be $2.67 \mathrm{~ns}$. After the interpolation, the time delay $(t)$ is calculated by the cross-correlation function. The calculated time delay and error rates are shown in Table 3; in this table, the "Method 1" represents the proposed fuzzy interpolation cross-correlation method and "Method 2" denotes the cubic spline interpolation crosscorrelation method.

In order to verify the practicability of the proposed fuzzy interpolation cross-correlation method for improving the accuracy of the time-delay estimation of PD UHF pulse signals, another verification experiment is performed in the laboratory. And the equipment arrangement is similar to
Figure 2. The received PD UHF signals are interpolated by one point in adjacent sampling points using fuzzy interpolation based on the evolved fuzzy rule base; this is the sampling rate after interpolation is $2 \mathrm{GHz}$. The results are shown in Figures 6(a) 6(e). These figures show that the interpolated PD UHF pulse signals curved by fuzzy interpolation are smoother than those before interpolation. From Figures 6(e) and 6(f), the maximum correlation coefficient happens as 6 translation points and 4 translation points, respectively. That is, the calculated time delays are $6 \mathrm{~ns}$ and $2 \mathrm{~ns}$ for fuzzy interpolation inference and cubic spline interpolation, respectively. Thus, the error rates are $124 \%$ and $25.1 \%$. With the increase of the interpolated point in adjacent sampling points, the error rate gradually decreases, as shown in Figure 7.

By analyzing the calculated time delay and the error rate, the following conclusions can be drawn:

(1) The time-delay calculation results are the multiple of the sampling interval; however, in most cases of the actual PD detection, the time delay is not an integer multiple of the sampling interval, which will lead to 


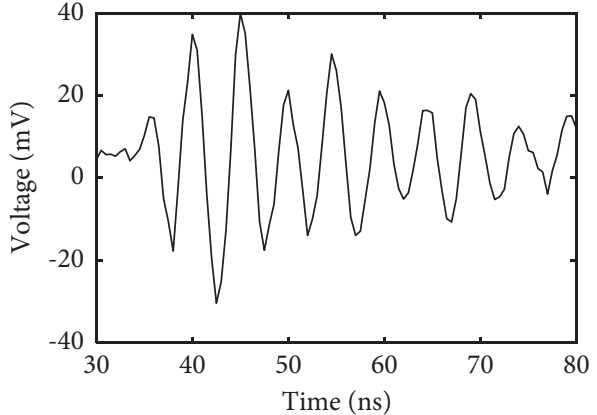

(a)

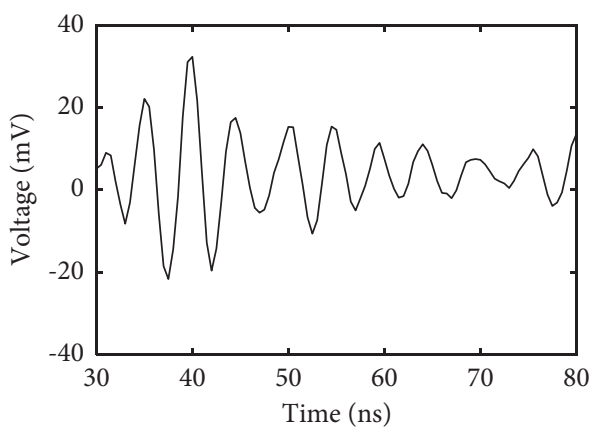

(c)

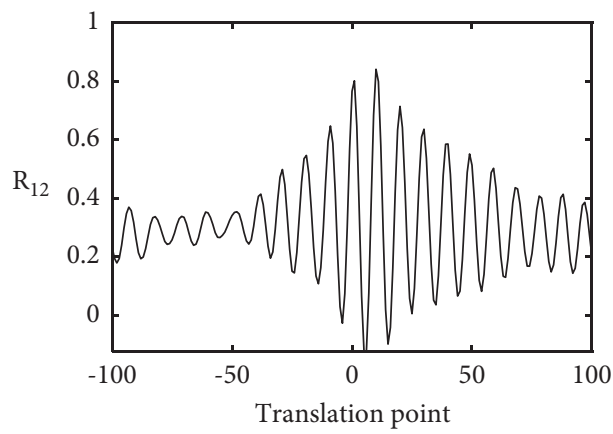

(e)

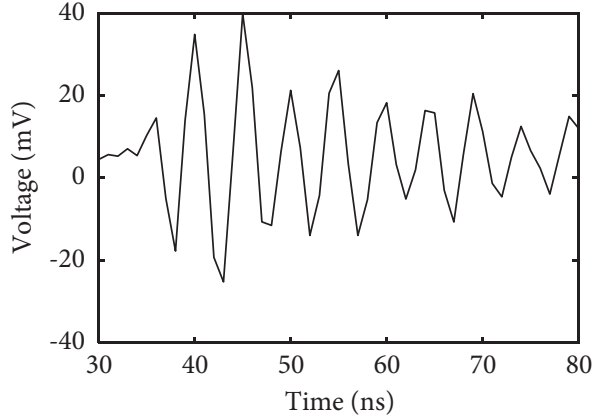

(b)

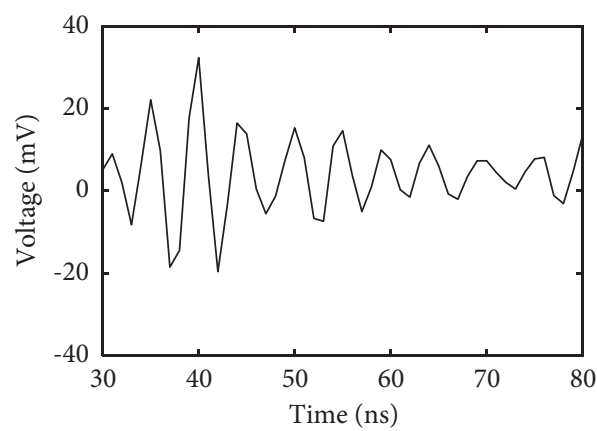

(d)

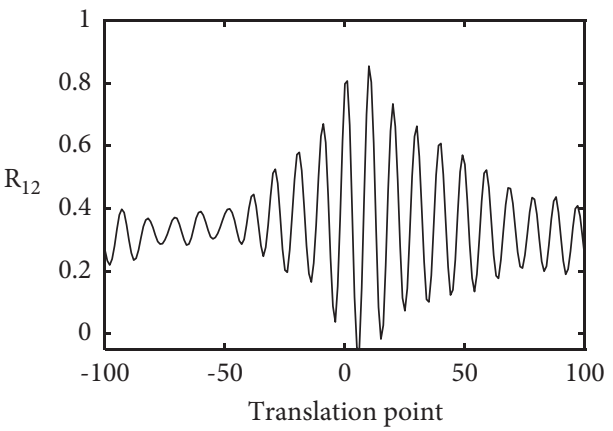

(f)

FIgURE 5: The interpolated $2 \mathrm{GHz}$ PD UHF pulse signals and the correlation coefficient curve. (a) Interpolated antenna A received PD signal by fuzzy interpolation. (b) Interpolated antenna A received PD signal by cubic spline interpolation. (c) Interpolated antenna B received PD signal by fuzzy interpolation. (d) Interpolated antenna B received PD signal by cubic spline interpolation. (e) Correlation coefficient curve for interpolated signals by fuzzy interpolation. (f) Correlation coefficient curve for interpolated signals by cubic spline interpolation.

TABLE 3: The calculated time delay and errors.

\begin{tabular}{|c|c|c|c|c|c|c|}
\hline \multirow{2}{*}{ Interpolating point } & \multirow{2}{*}{ Sampling rate $(\mathrm{GHz})$} & \multicolumn{3}{|c|}{ Time delay (ns) } & \multicolumn{2}{|c|}{ Error rate $(\%)$} \\
\hline & & Expected value & Method 1 & Method 2 & Method 1 & Method 2 \\
\hline 0 & 1 & 2.67 & 5 & 5 & 87.3 & 87.3 \\
\hline 1 & 2 & 2.67 & 3.5 & 4 & 31.1 & 49.8 \\
\hline 3 & 4 & 2.67 & 3.25 & 3.75 & 21.7 & 40.4 \\
\hline 7 & 8 & 2.67 & 2.75 & 3.0 & 3.0 & 12.4 \\
\hline 15 & 16 & 2.67 & 2.75 & 2.88 & 3.0 & 7.87 \\
\hline
\end{tabular}

* "Method 1" represents the proposed fuzzy interpolation cross-correlation method and "Method 2" represents denotes the cubic spline interpolation crosscorrelation method. 


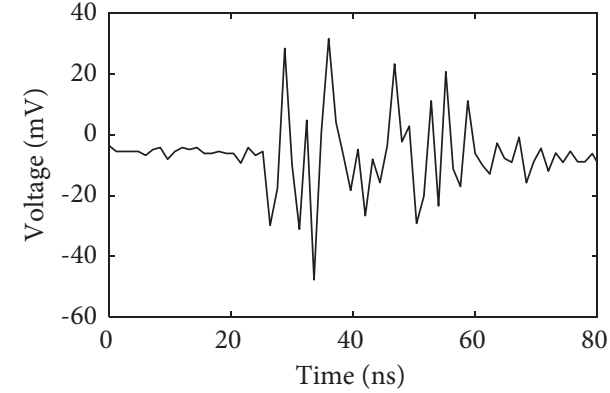

(a)

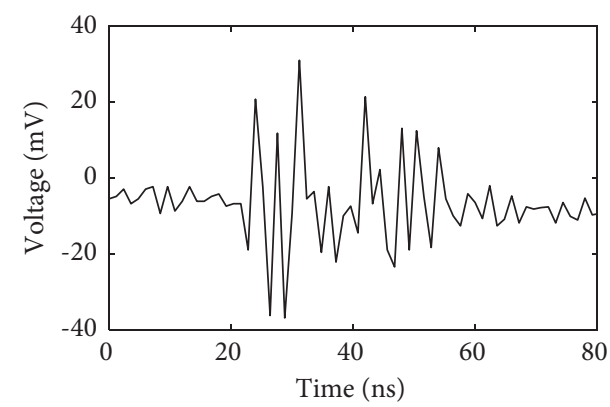

(c)

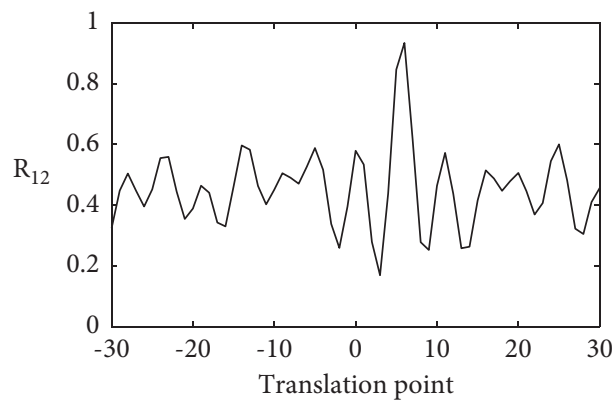

(e)

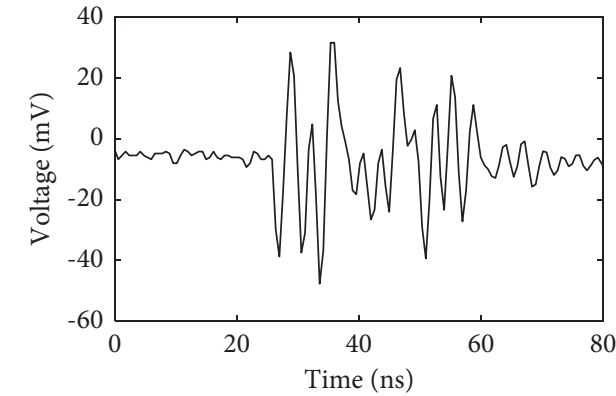

(b)

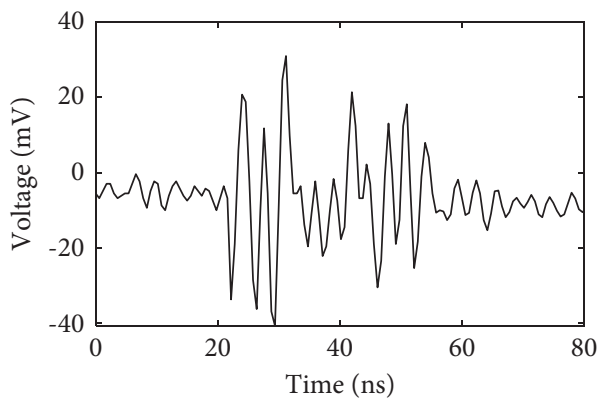

(d)

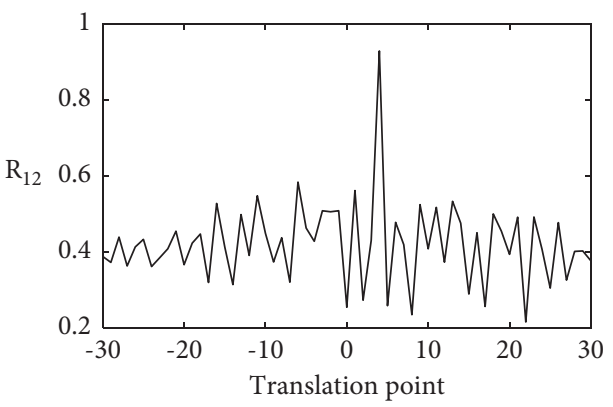

(f)

FIgURE 6: The verification experiment results. (a) Antenna A received PD UHF signals. (b) Interpolated antenna A received PD signal by fuzzy interpolation. (c) Antenna B received PD UHF signals. (d) Interpolated antenna A received PD signal by fuzzy interpolation. (e) Correlation coefficient curve for received signals. (f) Correlation coefficient curve for interpolated signals by fuzzy interpolation.

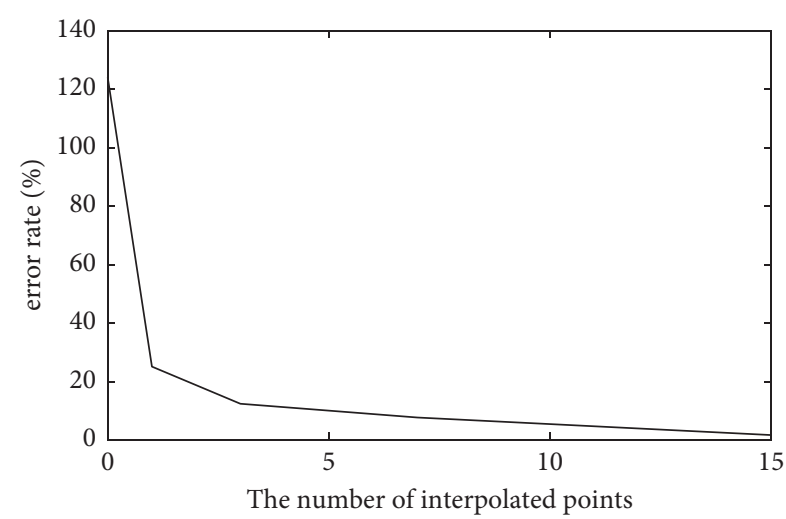

Figure 7: The error rate of the interpolated point in adjacent sampling points.

the estimation error. The proposed fuzzy interpolation cross-correlation method can improve the sampling rate and reduce the sampling interval, which will improve the time-delay estimation accuracy.

(2) At the same sampling rate, the proposed fuzzy interpolation cross-correlation method has greater time-delay estimation accuracy than the cubic spline interpolation cross-correlation method. The minimum error rate is $3.0 \%$. The reason is that the fuzzy rule base can express the PD feature information.

(3) As the number of interpolation points reaches a certain amount (it is 7 points in this experiment), the further improvement of the interpolation points does not improve the accuracy of the time-delay estimation accuracy.

\section{Conclusion}

Time-delay estimation of PD UHF pulse signals plays a key role in detecting the PD location. This paper proposes a fuzzy interpolation cross-correlation method for PD UHF pulse 
signal time-delay estimation. The proposed method consists of two key components, including fuzzy interpolation inference improving the PD UHF pulse signals sampling rate and the cross-correlation algorithm used to estimate the time delay. The comparative experimental results based on different interpolation points demonstrated the power of the proposed method in improving the accuracy of the PD UHF pulse signals time-delay estimation, and the minimum error rate is $3.0 \%$. It is not easy to improve the hardware sampling rate of the PD detection system in the practical application; the proposed fuzzy interpolation cross-correlation method should be an effective approach to improving the time-delay estimation with the lower sampling rate in the practical PD detection, and it is of high pragmatic and financial interest towards the PD detection system.

\section{Data Availability}

The data used to support the findings of this study are included within the article.

\section{Conflicts of Interest}

The authors declare that they have no conflicts of interest.

\section{Acknowledgments}

This work was supported by the Science and Technology Project of State Grid Tianjin Electric Power Company in 2021 (no. KJ21-1-9).

\section{References}

[1] H. Chai, B. T. Phung, and S. Mitchell, "Application of UHF sensors in power system equipment for partial discharge detection: a review," Sensors, vol. 19, no. 5, pp. 1029-1051, 2019.

[2] P. Romano, G. Presti, A. Imburgia, and R. Candela, "A new approach to partial discharge detection under DC voltage," IEEE Electrical Insulation Magazine, vol. 34, no. 4, pp. 32-41, 2018.

[3] H. Jahangir, A. Akbari, P. Werle, and J. Szczechowski, "UHF PD measurements on power transformers-advantages and limitations," IEEE Transactions on Dielectrics and Electrical Insulation, vol. 24, no. 6, pp. 3933-3940, 2018.

[4] J. Liu, J. Tang, and Y. Lang, "Approach for timedelay location of UHF signals of GIS partial discharge based on interpolating cross-correlation function," Gaoya Dianqi/High Voltage Apparatus, vol. 54, pp. 62-67, 2018.

[5] X. Rao, K. Zhou, Y. Li, G. Zhu, and P. Meng, "A new crosscorrelation algorithm based on distance for improving localization accuracy of partial discharge in cables lines," Energies, vol. 13, no. 17, pp. 4549-4561, 2020.

[6] T. Jin, Q. Li, and M. A. Mohamed, "A novel adaptive EEMD method for switchgear partial discharge signal denoising," IEEE Access, vol. 7, pp. 58139-58147, 2019.

[7] X. Chen, Y. Qian, Y. Xu, G. Sheng, and X. Jiang, "Energy estimation of partial discharge pulse signals based on noise parameters," IEEE Access, vol. 4, pp. 10270-10279, 2017.

[8] H. D. O. Mota, F. H. Vasconcelos, and C. L. de Castro, "A comparison of cycle spinning versus stationary wavelet transform for the extraction of features of partial discharge signals," IEEE Transactions on Dielectrics and Electrical Insulation, vol. 23, no. 2, pp. 1106-1118, 2016.

[9] H. Lu, Y. Liu, Z. Fei, and C. Guan, "An outlier detection algorithm based on cross-correlation analysis for time series dataset," Ieee Access, vol. 6, pp. 53593-53610, 2018.

[10] D. Dukanac, "Application of UHF method for partial discharge source location in power transformers," IEEE Transactions on Dielectrics and Electrical Insulation, vol. 25, no. 6, pp. 2266-2278, 2018.

[11] L. Tang, Y. Hu, H. Wang, Y. Yang, and X. Jiang, “Time-delay estimation of partial discharge UHF pulse signals based on interpolation cross-relation algorithm," High Voltage Engineering, vol. 41, no. 10, pp. 3320-3325, 2015.

[12] A. Jafarian, R. Jafari, M. Mohamed Al Qurashi, and D. Baleanu, "A novel computational approach to approximate fuzzy interpolation polynomials," SpringerPlus, vol. 5, no. 1, pp. 1428-1511, 2016.

[13] N. Naik, D. Ren, and Q. Shen, "Dynamic fuzzy rule interpolation and its application to intrusion detection," IEEE Transactions on Fuzzy Systems, vol. 26, no. 4, pp. 1878-1892, 2017.

[14] L. Yang, J. Li, F. Chao, P. Hackney, and M. Flanagan, "Job shop planning and scheduling for manufacturers with manual operations," Expert Systems, vol. 38, no. 7, Article ID e12315, 2018.

[15] K. Yin, K. Xiang, M. Pang, J. Chen, P. Anderson, and L. Yang, "Personalised control of robotic ankle exoskeleton through experience-based adaptive fuzzy inference," IEEE Access, vol. 7, pp. 72221-72233, 2019.

[16] Y. Jiang, S. Jin, and J. Peng, "Hierarchical fuzzy rule interpolation and its application for hotels location selection," International Journal of Cognitive Informatics and Natural Intelligence, vol. 10, no. 1, pp. 55-79, 2016.

[17] T. Chen, C. Shang, J. Yang, F. Li, and Q. Shen, "A new approach for transformation-based fuzzy rule interpolation," IEEE Transactions on Fuzzy Systems, vol. 28, no. 12, pp. 3330-3344, 2019. 